Preprint de "Stability of matchings when individuals have preferences over colleagues," de Bhaskar Dutta i Jordi Massó. Journal of Economic Theory 75, 464-475 (1997). Lliurat a Elsevier el gener de 1997. 


\title{
Stability of Matchings When Individuals Have Preferences Over Colleagues*
}

\author{
by \\ Bhaskar Dutta $^{\dagger}$ and Jordi Massó
}

Revised: January, 1997

${ }^{*}$ We thank David Pérez-Castrillo, Alvin Roth and an anonimous referee for helpful comments. Financial support from DGICYT (PB92-0590) and CIRIT (GRQ93-2044) research projects are aknowledged.

${ }^{\dagger}$ Indian Statistical Institute. 7 SJS Sansanwal Marg. New Delhi 110016, India.

†.Departament d’Economia i d'Història Econòmica and CODE. Universitat Autònoma de Barcelona. 08193, Bellaterra (Barcelona), Spain. 
Running head: Preferences Over Colleagues

\title{
Proofs should be send to:
}

\author{
Jordi Massó \\ Departament d'Economia i d'Història Econòmica \\ Universitat Autònoma de Barcelona \\ 08193, Bellaterra (Barcelona), Spain
}




\begin{abstract}
In the standard two-sided matching models, agents on one side of the market (the institutions) can each be matched to a set of agents (the individuals) on the other side of the market, and the individuals only have preferences defined over institutions to which they can be matched. We explicitly study the consequences for stability when the composition of one's coworkers or colleagues can affect the preferences over institutions. Journal of Economic Literature Classification Number: J41.
\end{abstract}




\section{Introduction}

A large class of two-sided matching models describe situations in which agents on one side of the market, say firms or colleges or more generally institutions, are each "matched" to agents, say workers or students or individuals, on the other side of the market. ${ }^{1}$ A common assumption in these many-to-one matching models is that individuals have preferences defined only over the institutions to which they can be matched, although the special problems posed by couples was recognized. As Roth and Sotomayor [6, page 171] remarked, "we continue to make the simplifying assumption that workers are indifferent to which other workers are employed by the same firm."

A moment's reflection is enough to convince us that there are many instances where this "simplifying assumption" is unlikely to hold good. For instance, university professors care about the composition of the rest of the faculty, while soccer players would prefer to join a team of Peles and Maradonas. Clearly, the composition of one's coworkers or colleagues can affect the preferences over institutions. The purpose of this paper is to incorporate workers' preferences over matchings which depend on the composition of colleagues into the traditional theory of twosided matching models. In particular, we analyse the consequences of imposing plausible restrictions on individuals' preferences over (institution-colleagues) pairs.

We essentially assume that workers' preferences are lexicographic. Within this broad category, one possibility is to assume that although workers care about who their coworkers are, it is their preferences over firms which dictate their overall preferences over firm-colleague pairs. We show that when workers preferences are of this type, then the set of matchings in the $\operatorname{core}^{2}$ is nonempty.

We then go on to examine whether the set of matchings in the core remains nonempty when workers' preferences over colleagues dictate their overall preferences. Within this class of "worker-lexicographic" preferences, we impose further restrictions. We first consider the case when a subset of the individuals are couples. We assume that each couple prefers a matching in which they are matched together with an institution rather than a matching in which they are paired with different institutions, irrespective of the "quality" of the institution. We

\footnotetext{
${ }^{1}$ Crawford and Knoer [1], Kelso and Crawford [3], Roth [4, 5] are a small sample of this literature. See Roth and Sotomayor [6] for an illuminating and comprehensive survey of this literature as well as an exhaustive bibliography.

${ }^{2}$ Throughout this paper, we assume that firms' preferences over sets of workers satisfy a condition called substitutability. This condition is assumed even in the traditional model, where it turns out to be sufficient for a nonempty core.
} 
show that despite the presence of couples, the set of stable matchings remains nonempty when preferences satisfy a condition similar to substitutability.

We then go on to assume that all workers share a common opinion about the relative desirability of all workers. In other words, there is a unanimous ranking of all workers, and any worker prefers to join a set of workers containing higher-ranked workers. An alternative assumption is that workers' preferences are

separable. So, each worker divides his or her set of potential colleagues into the set of good and bad workers. Adding a good worker leads to a better set, while adding a bad worker leads to a worse set.

However, it turns out that in both the latter cases of worker-lexicographic preferences, one can construct preference profiles which result in the core being empty. Hence, this paper shows that one of the major results of the standard model - namely, the existence of matchings which are immune to blocking by a coalition of firms and agents - is not particularly robust.

\section{Notation and Definitions}

The agents in our market consist of a set $\mathcal{F}$ of $n$ firms, and a set $\mathcal{W}$ of $p$ workers. Generic elements of $\mathcal{W}$ will be denoted by $w_{i}, m_{i}, w, i, j$, etc., while those of $\mathcal{F}$ will be denoted by $F_{i}, F_{j}, F$, etc. In general, $\mathcal{F}$ represents the set of institutions (firms, universities, research establishments), while $\mathcal{W}$ is the set of individuals (workers, university professors, researchers). We will typically use the terms firms and workers to represent institutions and individuals.

Firms hire sets of workers, and each $F_{j} \in \mathcal{F}$ has a strict preference ordering $P\left(F_{j}\right)$ over $2^{\mathcal{W}} \cup\left\{F_{j}\right\}$, where $2^{\mathcal{W}}$ is the set of all nonempty subsets of $\mathcal{W}$. For any $w_{i} \in \mathcal{W}$, let $\mathcal{W}_{i}=\left\{S \mid S \subseteq \mathcal{W}, w_{i} \in S\right\}$. Each worker $w_{i}$ has a preference ordering $R\left(w_{i}\right)$ defined over $\left(\mathcal{F} \times \mathcal{W}_{i}\right) \cup\left\{w_{i}\right\}$ with asymmetric component $P\left(w_{i}\right)$. Note that this formulation allows a worker to care about the firm that she is matched with, as well as with her coworkers.

A matching will be a particular assignment of workers to firms keeping the bilateral nature of their relationship, as well as the possibility of any particular agent(s) being unable to find partners. The formal definition is given below.

Definition 1: A matching $\mu$ is a mapping from $\mathcal{F} \cup \mathcal{W}$ into the set of all nonempty subsets of $\mathcal{F} \cup \mathcal{W}$ such that for all $w \in \mathcal{W}$ and $F \in \mathcal{F}$ :

(i) $|\mu(w)|=1$ and $\mu(w)=w$ if $\mu(w) \notin \mathcal{F}$;

(ii) $\mu(F) \subseteq \mathcal{W} \cup\{F\}$, and $\mu(F)=F$ if $\mu(F) \notin 2^{\mathcal{W}}$; 
(iii) $\mu(w)=F$ iff $w \in \mu(F)$.

Given any matching $\mu$, and any worker $w$ matched at $\mu$, let $F=\mu(w)$ and $S=\mu(F)$. Then, we will represent $S$ as $\mu^{2}(w)$. That is, $\mu^{2}(w)$ is the set consisting of worker $w$ and her colleagues in the firm with which she is matched.

Let $F \in \mathcal{F}$. Then, the preference ordering $P(F)$ of firm $F$ over $2^{\mathcal{W}} \cup\{F\}$ induces an ordering over the set of matchings. Thus, firm $F$ prefers $\mu$ to $\bar{\mu}$ if $\mu(F) P(F) \bar{\mu}(F)$. Similarly, the preference ordering $P(w)$ of a worker $w$ induces an ordering over the set of matchings. So, worker $w$ prefers $\mu$ to $\bar{\mu}$ if $\left(\mu(w), \mu^{2}(w)\right) P(w)\left(\bar{\mu}(w), \bar{\mu}^{2}(w)\right){ }^{3}$ With some abuse of notation, we will also let $P(F)$ and $P(w)$ denote the induced orderings of $F$ and $w$ over the set of matchings.

Definition 2: A matching $\mu$ is individually rational if for all $F \in \mathcal{F}$ and $w \in \mathcal{W}$, not $\{F\} P(F) \mu(F)$ and $\left(\mu(w), \mu^{2}(w)\right) R(w)\{w\}$.

So, a matching is individually rational if no worker or firm prefers to be unmatched. Notice that in a framework where workers have preferences over potential colleagues, this definition corresponds to the usual interpretation of individual rationality as a constraint which expresses what an individual agent can achieve unilaterally. In contrast, a matching $\mu$ in the traditional model is defined to be individually rational only if no firm $F$ prefers any subset of $\mu(F)$ to $\mu(F)$. Roth and Sotomayor [6] remark that "this recognises that $F$ may fire some workers in $\mu(F)$ if it chooses, without affecting other members (italics ours) of $\mu(F) . "$ Obviously, when workers have preferences over potential colleagues, if a subset of $\mu(F)$ is fired, then some of the remaining workers may well quit. This makes our definition of individual rationality more appropriate in the present framework.

Definition 3: Given any profile of preferences $\mathbf{P}=\left(\{R(w)\}_{w \in \mathcal{W}},\{P(F)\}_{F \in \mathcal{F}}\right)$, a matching $\mu$ is in the core, denoted $C(\mathbf{P})$, if there is no $A \subseteq \mathcal{F} \cup \mathcal{W}$ and a matching $\mu^{\prime}$ such that:

(i) $\mu^{\prime}(w) \in A \quad \forall w \in A$;

(ii) $\mu^{\prime}(F) \subseteq A \quad \forall F \in A$;

(iii) $\mu^{\prime} P(F) \mu \quad \forall F \in A$;

(iv) $\mu^{\prime} P(w) \mu \quad \forall w \in A$.

Remark 1: If such an $A$ and $\mu^{\prime}$ exist, then we will say that $\mu$ is blocked by $A$.

\footnotetext{
${ }^{3}$ If the worker $w$ is unmatched according to either $\mu$ or $\bar{\mu}$, then appropriate changes need to be made.
} 
Also, note that if a matching is not individually rational, then it is obviously not in the core.

So, a matching $\mu$ is in the core if no group of firms and workers can obtain a more preferred matching entirely on their own.

Remark 2: An alternate version of the core, denoted by $C^{W}(\mathbf{P})$, is the set of matchings which cannot be "weakly blocked" by any group of firms and workers, where $\mu$ is weakly blocked by $A$ via $\mu^{\prime}$ if all members of $A$ find $\mu^{\prime}$ at least as good as $\mu$ and at least one member of $A$ strictly prefers $\mu^{\prime}$ to $\mu$.

Remark 3: In the definition of the core given above, a matching may not be in the core because it is blocked by a group of firms and some set of workers. However, it is easy to show that if a matching is not in the core, then either it is not individually rational or it is blocked by a single firm and some workers.

\section{Preference Restrictions}

This section contains a description of various alternative restrictions that will be imposed on workers' preferences. However, we first define a restriction of substitutability which will be imposed on firms' preferences. It is known that when firms' preferences are substitutable, the core is nonempty in the standard or traditional model when workers are indifferent about their coworkers. Since our purpose is to examine the consequences of permitting workers to care about their coworkers, we will assume that firms' preferences are substitutable.

Given any set $S \subseteq \mathcal{W}$, let $C h_{F}(S)$ denote firm $F^{\prime}$ s most-preferred subset of $S$ according to its preference ordering $P(F)$. Since $F$ is not a subset of $S \subseteq \mathcal{W}$, we are identifying the empty set with $F$ itself in its preference ordering.

Definition 4: $P(F)$ has the property of substitutability if for any set $S$ containing workers $w, w^{\prime} \in \mathcal{W}\left(w \neq w^{\prime}\right), w \in C h_{F}(S)$ implies $w \in C h_{F}\left(S \backslash\left\{w^{\prime}\right\}\right)$.

So, if $F$ has substitutable preferences, then it regards workers in $C h_{F}(S)$ as substitutes rather than complements since it continues to want to employ worker $w$ even if some of the other workers become unavailable.

Let $\mathcal{P}_{T}$ denote the set of all logically possible preference profiles where firms' preferences are substitutable and workers' preferences correspond to the tradi- 
tional model. ${ }^{4}$

We first consider markets in which a subset of individuals consists of couples. Let $\mathcal{W}=\mathcal{W}^{c} \cup Q$, where $\mathcal{W}^{c}$ is the set of workers who are couples, and $Q$ is the set of single workers. We will sometimes find it convenient to represent a typical couple $(m, w)$ as $c$, and $C$ as the set of couples.

We will assume that any member of a couple always prefers to be matched together with his or her partner, rather than be matched alone. So, consider any $c=(m, w)$. Then, let $\mathcal{W}_{\{c\}}=\mathcal{W}_{m} \cap \mathcal{W}_{w}$, that is $\mathcal{W}_{\{c\}}$ is the subsets of $\mathcal{W}$ containing both $m$ and $w$.

Definition 5: Let $i$ be any member of some couple $c=(i, j) \in C$. Then, $i$ 's preference ordering, $P(i)$ satisfies Togetherness if:

(i) for all pairs $(F, S),\left(F^{\prime}, S^{\prime}\right) \in \mathcal{F} \times \mathcal{W}_{i},(F, S) P(i)\left(F^{\prime}, S^{\prime}\right)$ whenever $S \in \mathcal{W}_{\{c\}}$ and $S^{\prime} \notin \mathcal{W}_{\{c\}}$;

(ii) for all $F \in \mathcal{F}$ and $S, S^{\prime} \in \mathcal{W}_{\{c\}}, i$ is indifferent between $(F, S)$ and $\left(F, S^{\prime}\right)$;

(iii) for all $F \in \mathcal{F}$ and $S \in \mathcal{W}_{i}$ such that $j \notin S, i$ is indifferent between $(F,\{i\})$ and $(F, S)$;

(iv) for all distinct $F, F^{\prime} \in \mathcal{F}$ and $S, S^{\prime} \in \mathcal{W}_{i}$, either $(F, S) P(i)\left(F^{\prime}, S^{\prime}\right)$ or $\left(F^{\prime}, S^{\prime}\right) P(i)(F, S)$.

We will assume a stronger form of substitutability, which we call group substitutability guaranteeing that there are no complementarities among groups of workers. Denote by $W$ the family of sets of the form $S=\left\{S_{1}, \ldots, S_{K}\right\}=\bigcup_{k \in \mathcal{K}} S_{k}$, where for each $k \in \mathcal{K}=\{1, \ldots, K\}$, the set $S_{k}$ is either an element of $\mathcal{W}^{c}, Q$, or $C$. Obviously, every element of $\mathcal{W}$ can show up at most once in the set $S$. Consider firm $F$ with preferences $P(F)$ over all subsets of $W$, and consider any set $S=\left\{S_{1}, \ldots, S_{K}\right\}$. Let $C h_{F}^{*}(S)=\left\{T \in S \cup F \mid T P(F) \bigcup_{k \in M} S_{k}\right.$ for all $\left.M \subseteq \mathcal{K}\right\}$ denote firm $F$ 's most-preferred subset of $S$ according to its preference ordering $P(F)$.

Definition 6: $P(F)$ has the property of group substitutability if for all $S=$ $\left\{S_{1}, \ldots, S_{K}\right\}=\bigcup_{k \in \mathcal{K}} S_{k} \in W$, every pair $S_{k}, S_{k^{\prime}} \in S, S_{k} \neq S_{k^{\prime}}, S_{k} \in C h_{F}^{*}(S) \Rightarrow$ $S_{k} \in C h_{F}^{*}\left(S \backslash S_{k^{\prime}}\right)$.

Let $\mathcal{P}_{T}^{c}$ denote the set of preference profiles where preferences of individuals in $\mathcal{W}^{c}$ satisfy togetherness, preferences of institutions satisfy group substitutability,

\footnotetext{
${ }^{4}$ That is, workers are indifferent about who their coworkers are, but have a strict preference ordering over $\mathcal{F}$.
} 
while those of single individuals conform to those of $\mathcal{P}_{T}$.

Note that we have modelled preferences of couples in a different way from that of Roth and Sotomayor [6], who assume that a couple have a single preference ordering over pairs of firms. This corresponds to situations where couples do not mind being matched to firms which are geographically close to each other. Our formulation implicitly assumes that the option of being matched to firms sufficiently close to each other is not present.

Apart from the markets with couples, we are going to assume that each worker $w_{i}$ 's preferences over $\mathcal{F} \times \mathcal{W}_{i}$ are lexicographic. Obviously, when workers' preferences are lexicographic, their preferences for either firms or coworkers could dominate their overall preference ordering. Thus, we have two kinds of lexicographic preferences. These are defined below.

Definition 7: Worker $w_{i}$ 's preferences are $\mathcal{F}$-lexicographic if there is a strict ordering $P_{i}$ over $\mathcal{F}$ such that for all $(F, S),\left(F^{\prime}, S^{\prime}\right) \in \mathcal{F} \times \mathcal{W}_{i},(F \neq$ $\left.F^{\prime}\right),(F, S) P\left(w_{i}\right)\left(F^{\prime}, S^{\prime}\right) \Leftrightarrow F P_{i} F^{\prime}$, and $(F, S) P\left(w_{i}\right) w_{i} \Leftrightarrow F P\left(w_{i}\right) w_{i}$.

Definition 8: Worker $w_{i}$ 's preferences are $\mathcal{W}$-lexicographic if there is a strict ordering $\bar{P}_{i}$ over $\mathcal{W}_{i}$ such that for all $(F, S),\left(F^{\prime}, S^{\prime}\right) \in \mathcal{F} \times \mathcal{W}_{i},(S \neq$ $\left.S^{\prime}\right),(F, S) P\left(w_{i}\right)\left(F^{\prime}, S^{\prime}\right) \Leftrightarrow S \bar{P}_{i} S^{\prime}$.

Thus, if $w_{i}$ 's preferences are $\mathcal{F}$-lexicographic, then $w_{i}$ 's ranking of firms, $P_{i}$, determines $w_{i}$ ' preference ordering over all (firm, coworkers) pairs in which firms are distinct. $\mathcal{W}$-lexicographic preferences have an analogous interpretation. We denote by $\mathcal{P}_{\mathcal{F}}$ the set of all logically possible preference profiles where workers' preferences are $\mathcal{F}$-lexicographic and firms' preferences are substitutable.

We impose additional restrictions when workers' preferences are $\mathcal{W}$ lexicographic. When preferences are $\mathcal{W}$-lexicographic, it is sufficient to describe restrictions which operate on workers' rankings over sets of coworkers. Consider, for instance, the market for economists. Suppose all economists have a unanimous ranking of economists according to their desirability. Since there are obvious externalities generated by faculty members, most economists would prefer to join a faculty consisting of higher ranked economists. This provides the motivation for the next definition.

Definition 9: Workers' preference orderings satisfy unanimous ranking according to desirability (URD) if $\forall w_{i} \in \mathcal{W}, \forall S, T \in \mathcal{W}_{i}$ such that $S=$ $\left(T \cup\left\{w_{j}\right\}\right) \backslash\left\{w_{k}\right\}, w_{j} \notin T$, and $w_{k} \in T$, we have that $S \bar{P}_{i} T$ iff $j<k$. 
Thus, all workers agree that $w_{j}$ is a "better" worker than $w_{j+1}$ and their preferences over coworkers respond to this ranking.

Remark 4: Note that we are not going to assume that a firm's preference ordering over sets of workers is consistent with this unanimous ranking of workers. Suppose, for instance, that a higher-ranked worker commands a higher salary than a lowerranked worker. If the salary differential is large enough, then the net benefit generated by the higher-ranked worker may well be lower.

Let $\mathcal{P}_{U R D}$ be the set of all preference profiles such that workers' preference orderings are $\mathcal{W}$-lexicographic and which satisfy $U R D$, while firms' preferences are substitutable.

An alternative restriction will be one of separability. That is, each $w_{i} \in \mathcal{W}$ divides $\mathcal{W} \backslash\left\{w_{i}\right\}$ into the set of good and bad workers. Moreover, adding a good worker leads to a better set, while adding a bad worker leads to a worse set.

Definition 10: A worker $w_{i}$ 's preference ordering satisfies separability if there is a partition $\left\{G_{i}, B_{i}\right\}$ of $\mathcal{W} \backslash\left\{w_{i}\right\}$ such that for all $S \in \mathcal{W}_{i}$ and $w_{j} \notin S$,

$$
\left(S \cup\left\{w_{j}\right\}\right) \bar{P}_{i} S \quad \text { iff } \quad w_{j} \in G_{i} .
$$

Remark 5: Note that workers do not necessarily agree on which workers are good and bad.

Let $\mathcal{P}_{S}$ be the set of all profiles such that workers' preferences satisfy separability, while firms preferences are substitutable.

\section{The Results}

In this section, we explore the consequences of the various restrictions on preferences introduced in the previous section.

First, we show that the set of matchings in the core of the market with couples is nonempty when preferences profiles are in $\mathcal{P}_{T}^{c} .^{5}$ In order to prove this result, we need to modify the deferred-acceptance algorithm, which was originally defined by Gale and Shapley [2].

\footnotetext{
${ }^{5}$ Roth and Sotomayor [6] show that under their formulation of preferences of couples, the set of matchings in the core may be empty.
} 
We describe the version of the algorithm in which individuals make offers to firms. At any step of the algorithm, an individual (any worker) makes an offer to its most-preferred firm ${ }^{6}$ from amongst the set of firms who have not already rejected the worker, while a firm rejects all those workers who are not in the firm's choice set from those proposals it has not yet rejected. The algorithm terminates when no firm rejects a worker. Since firms' preferences are substitutable, a firm never regrets the decision to reject a worker at any step.

Now, consider the following modification of this algorithm.

Stage 1: For all $c \in C$, let $P(c)$ denote the restriction of $P(w)$ on the set $\left(\mathcal{F} \times \mathcal{W}_{\{c\}}\right) \cup\{w\}$. Consider market $M^{1}$ where each $c \in C$ is treated as a single individual with preference ordering $P(c)$, so that the set of "individuals" is $C \cup Q$. The set of firms remains $\mathcal{F}$. Note that in $M^{1}$, preferences of all agents satisfy the assumptions of the traditional model, since conditions (ii) and (iv) in the definition of togetherness holds and firms have group substitutable preferences.

Now, use the deferred-acceptance algorithm with workers proposing, and let $\mu_{1}$ be the resulting matching. Let $C^{1}$ be the set of couples who are matched to some firm in $\mathcal{F}$. If $C^{1}=C$, then stop the algorithm. Otherwise, go to Stage 2 .

Stage 2: For all $(m, w)=c \in C \backslash C^{1}$, let $\bar{P}(m)$ and $\bar{P}(w)$ denote the restriction of $P(m)$ and $P(w)$ on $(\mathcal{F} \times\{m\}) \cup\{m\}$ and $(\mathcal{F} \times\{w\}) \cup\{w\}$ respectively. Let $M^{2}$ denote the market where each $c \in C^{1}$ is treated as a single individual with preference $P(c)$, while $\bar{P}(m)$ and $\bar{P}(w)$ are the preferences of each pair $(m, w) \in$ $C \backslash C^{1}$. Each $i \in Q$ has the "original" preference ordering $P(i)$. Again, now by conditions (iii) and (iv) in the definition of togetherness and group substitutability, $M^{2}$ satisfies all the assumptions of the traditional model.

Let $\mu_{2}$ denote the matching resulting from the deferred-acceptance algorithm with workers proposing. Let $C^{2}$ denote the set of couples in $C^{1}$ who are matched to firms according to $\mu_{2}$. If $C^{1}=C^{2}$, then stop the algorithm.

In general, stop the algorithm in any stage $K$ such that $C^{K}=C^{K-1}$, and call $\mu_{K}$ the outcome produced by the algorithm.

Let us call this the multi-stage deferred-acceptance algorithm.

Theorem 1: Let $\mu^{*}$ be the outcome of the multi-stage deferred-acceptance algorithm. If $\mathbf{P} \in \mathcal{P}_{T}^{c}$ then $\mu^{*}$ is in the core of any market with couples.

Proof: Suppose $\mu^{*}$ is not in the core of the (original) market with couples. Since it is trivial to check that $\mu^{*}$ is individually rational, let $\mu^{*}$ be blocked by some

\footnotetext{
${ }^{6}$ Note that in the traditional model, individuals have a strict preference ordering over firms.
} 
pair $(F, S)$ where $S \subseteq \mathcal{W}$.

Let $\mu^{*}=\mu_{K}$, so that the multi-stage deferred-acceptance algorithm terminates in stage $K$. Note that by construction $C^{K}=C^{K-1}$.

We first show that $S \cap\left(C^{K-1} \cup Q\right)=\emptyset$. Obviously, since each $c \in C^{K-1}$ is matched to some firm in $\mu_{K}$, no member of $c$ would prefer a match with $F$ to $\mu_{K}$ if her partner is not matched to $F$.

So, suppose $(F, c) P(c)\left(\mu_{K}(c), c\right)$. Consider the deferred-acceptance algorithm in stage $K$. At some stage, $c$ must have made an offer to $F$, but was rejected. But, since $S P(F) \mu_{K}(F)$ and the firm's preference are group substitutable, $c$ cannot be contained in $S$.

For analogous reasons, $S \cap Q=\emptyset$.

Since couples in $C \backslash C^{K}$ "split up" in stage $K$ and since $\mu_{K}$ must be in the core of market $M^{K}$, the only remaining possibility is that $S$ consists of some couples in $C \backslash C^{K}$; that is, there are some $\left\{c_{1}, \ldots, c_{l}\right\}$ who are not matched as couples in $\mu_{K-1}$ (and hence $\mu_{K}$ ), but such that $F$ prefers these couples to $\mu_{K}(F)$.

Now, not $\mu_{K-1}(F) P(F) \mu_{K}(F)$. For, suppose $\mu_{K-1}(F) P(F) \mu_{K}(F)$. Choose $j \in \mu_{K-1}(F)$ such that $j \notin \mu_{K}(F)$. Then, $j$ made an offer to $F$ in some step $k$ in $M^{K-1}$ and was accepted. Given group substitutability, if $j$ made an offer to $F$ in $M^{K}$ in step $k$, then $j$ would be accepted by $F$. So, in $M^{K}, j$ is accepted by some firm $F^{\prime}$ in an earlier step $q$. Moreover, $F^{\prime}$ rejected $j$ in $M^{K-1}$. Again, this violates group substitutability of $P\left(F^{\prime}\right)$.

Hence, not $\mu_{K-1}(F) P(F) \mu_{K}(F)$. Note that $S P(F) \mu_{K}(F)$ implies $S P(F) \mu_{K-1}(F)$. Moreover, $S$ consists of couples who were not matched in $\mu_{K-1}$. Hence, $(F, S)$ blocks $\mu_{K-1}$, which contradicts the fact that $\mu_{K-1}$ is in the core of $M^{K-1}$.

In the next result, we will assume that workers' preferences are $\mathcal{F}$ lexicographic. We will see that in this case, the core is nonempty.

Let $\left(P\left(w_{1}\right), \ldots, P\left(w_{p}\right)\right)$ be any profile of $\mathcal{F}$-lexicographic workers' preferences. Let $P_{i}$ be the ordering over $\mathcal{F}$ induced by $P\left(w_{i}\right)$. Then, for all $\mathbf{P} \in \mathcal{P}_{F}$, let $\mathbf{P}^{t}=\left(\left\{P^{t}(F)\right\}_{F \in \mathcal{F}},\left\{P^{t}(w)\right\}_{w \in \mathcal{W}}\right)$ be the profile such that $P^{t}(F)=P(F)$ for all $F \in \mathcal{F}$, and $P^{t}\left(w_{i}\right)=P_{i}$ for all $w_{i} \in \mathcal{W}$. For any $\mathbf{P} \in \mathcal{P}_{F}$, we label $\mathbf{P}^{t}$ to be the induced traditional profile.

We remind the reader that $C^{W}\left(\mathbf{P}^{t}\right) \neq \emptyset .^{7}$

Theorem 2: For all $\mathbf{P} \in \mathcal{P}_{F}, C^{W}\left(\mathbf{P}^{t}\right) \subseteq C(\mathbf{P})$.

\footnotetext{
${ }^{7}$ See, for instance, Roth and Sotomayor [6, Proposition 5.36].
} 
Proof: Consider any $\mathbf{P} \in \mathcal{P}_{\mathcal{F}}$, let $\mathbf{P}^{t}$ be the induced traditional profile, and let $\mu \in C^{W}\left(\mathbf{P}^{t}\right)$.

Since $\mu \in C^{W}\left(\mathbf{P}^{t}\right), \mu$ is individually rational. Suppose $\mu \notin C(\mathbf{P})$. Then, $\mu$ is blocked by some pair $(F, S)$, where $F \in \mathcal{F}$ and $S \in 2^{\mathcal{W}}$. With some abuse of notation, we denote $\mu\left(w_{i}\right)=F_{i}$ for all $w_{i} \in \mathcal{W}$. So, $S P(F) \mu(F)$. Also, $(F, S) P\left(w_{i}\right)\left(F_{i}, \mu\left(F_{i}\right)\right) \forall w_{i} \in S$. The latter also implies that $F_{i} P_{i} F$ for $n o w_{i} \in S$. Hence, $(F, S)$ weakly blocks $\mu$ according to $\mathbf{P}^{t}$. This contradicts the hypothesis that $\mu \in C^{W}\left(\mathbf{P}^{t}\right)$.

We now analyse more "radical" departures from the traditional model.

Theorem 3: There is $\mathbf{P} \in \mathcal{P}_{U R D}$ such that $C(\mathbf{P})=\emptyset$.

Proof: Let $\mathcal{F}=\left\{F_{1}, F_{2}\right\}$, and $\mathcal{W}=\left\{w_{1}, w_{2}, w_{3}, w_{4}\right\}$.

We construct a preference profile $\mathbf{P} \in \mathcal{P}_{U R D}$ such that $C(\mathbf{P})=\emptyset$. Reminding the reader that workers' preferences are $\mathcal{W}$-lexicographic, we only describe workers' preferences over coworkers.

Let $\left\{w_{1}, w_{2}, w_{3}, w_{4}\right\}$ be the unanimous ranking of workers according to desirability; i.e., $w_{i}$ is ranked higher than $w_{i+1} . \mathbf{P}$ is given by the following table, where again, elements are ranked in descending order of preference and only acceptable partners are listed.

\begin{tabular}{cccccc}
$F_{1}$ & $F_{2}$ & $w_{1}$ & $w_{2}$ & $w_{3}$ & $w_{4}$ \\
\hline$\left\{w_{2}, w_{3}, w_{4}\right\}$ & $\left\{w_{1}, w_{2}\right\}$ & $\left\{w_{1}, w_{2}\right\}$ & $\left\{w_{1}, w_{2}, w_{3}\right\}$ & $\left\{w_{1}, w_{2}, w_{3}\right\}$ & $\left\{w_{1}, w_{4}\right\}$ \\
$\left\{w_{2}, w_{3}\right\}$ & $\left\{w_{1}, w_{4}\right\}$ & $\left\{w_{1}, w_{3}\right\}$ & $\left\{w_{1}, w_{2}, w_{4}\right\}$ & $\left\{w_{1}, w_{3}, w_{4}\right\}$ & $\left\{w_{2}, w_{4}\right\}$ \\
$\left\{w_{3}, w_{4}\right\}$ & $\left\{w_{1}\right\}$ & $\left\{w_{1}, w_{4}\right\}$ & $\left\{w_{2}, w_{3}, w_{4}\right\}$ & $\left\{w_{2}, w_{3}, w_{4}\right\}$ & $\left\{w_{1}, w_{3}, w_{4}\right\}$ \\
$\left\{w_{2}, w_{4}\right\}$ & $\left\{w_{2}\right\}$ & $\left\{w_{1}\right\}$ & $\left\{w_{1}, w_{2}\right\}$ & $\left\{w_{1}, w_{3}\right\}$ & $\left\{w_{2}, w_{3}, w_{4}\right\}$ \\
$\left\{w_{1}, w_{4}\right\}$ & $\left\{w_{3}\right\}$ & & $\left\{w_{2}, w_{3}\right\}$ & $\left\{w_{2}, w_{3}\right\}$ & $\left\{w_{3}, w_{4}\right\}$ \\
$\left\{w_{2}\right\}$ & $\left\{w_{4}\right\}$ & & $\left\{w_{2}, w_{4}\right\}$ & $\left\{w_{3}, w_{4}\right\}$ & $\left\{w_{4}\right\}$ \\
$\left\{w_{4}\right\}$ & & & $\left\{w_{2}\right\}$ & $\left\{w_{3}\right\}$ & \\
$\left\{w_{3}\right\}$ & & & & & \\
$\left\{w_{1}\right\}$ & & & & &
\end{tabular}

The reader can check that no worker can be unemployed in a matching $\mu$ if $\mu \in C(\mathbf{P})$. Now, individual rationality implies that the only candidates for a matching in $C(\mathbf{P})$ are: 


$$
\begin{array}{ccc} 
& F_{1} & F_{2} \\
\mu_{1} & \left\{w_{3}, w_{4}\right\} & \left\{w_{1}, w_{2}\right\} \\
\mu_{2} & \left\{w_{2}, w_{3}\right\} & \left\{w_{1}, w_{4}\right\} \\
\mu_{3} & \left\{w_{2}, w_{3}, w_{4}\right\} & \left\{w_{1}\right\}
\end{array}
$$

However, $\mu_{1}$ is blocked by $\left\{F_{1}\right\} \cup\left\{w_{2}, w_{3}, w_{4}\right\}$. Also, $\mu_{2}$ is blocked by $\left\{F_{2}\right\} \cup$ $\left\{w_{1}, w_{2}\right\}$ and $\mu_{3}$ is blocked by $\left\{F_{2}\right\} \cup\left\{w_{1}, w_{4}\right\}$.

Hence, $C(\mathbf{P})=\emptyset$.

In our next theorem, we show that the core can be empty even if workers' preferences are separable.

Theorem 4: There is $\mathbf{P} \in \mathcal{P}_{s}$ such that $C(\mathbf{P})=\emptyset$.

\begin{tabular}{|c|c|c|c|c|}
\hline$w_{1}$ & $w_{2}$ & $w_{3}$ & $w_{4}$ & $w_{5}$ \\
\hline$\left.w_{2}, w_{3}, w_{5}\right\}$ & $\left.w_{1}, w_{3}, w_{4}, w_{5}\right\}$ & $\left.w_{1}, w_{2}, w_{4}, w_{5}\right\}$ & $\left.w_{2}, w_{3}, w_{5}\right\}$ & $w_{1}, w_{2}, w_{3}$ \\
\hline
\end{tabular}

Proof: Let $\mathcal{F}=\left\{F_{1}, F_{2}\right\}$, and $\mathcal{W}=\left\{w_{1}, w_{2}, w_{3}, w_{4}, w_{5}\right\}$. Again, we construct a preference profile $\mathbf{P} \in \mathcal{P}_{S}$ such that $C(\mathbf{P})=\emptyset$.

First, the sets of workers judged to be good by each worker are shown below

The preference orderings, in descending order of preferences are:

\begin{tabular}{ccccc}
$F_{i}(i=1,2)$ & $w_{1}$ & $w_{2}$ & $w_{3}$ & $w_{5}$ \\
\hline$\left\{w_{2}, w_{3}, w_{4}, w_{5}\right\}$ & $\left\{w_{1}, w_{2}, w_{3}\right\}$ & $\left\{w_{2}, w_{4}, w_{5}\right\}$ & $\left\{w_{2}, w_{3}, w_{4}, w_{5}\right\}$ & $\left\{w_{1}, w_{5}\right\}$ \\
$\left\{w_{2}, w_{4}, w_{5}\right\}$ & $\left\{w_{1}, w_{5}\right\}$ & $\left\{w_{1}, w_{2}, w_{3}\right\}$ & $\left\{w_{1}, w_{2}, w_{3}\right\}$ & $\left\{w_{2}, w_{3}, w_{4}, w_{5}\right\}$ \\
$\left\{w_{1}, w_{2}, w_{3}\right\}$ & & $\left\{w_{2}, w_{3}, w_{4}\right\}$ & $\left\{w_{2}, w_{3}, w_{4}\right\}$ & \\
$\left\{w_{1}, w_{5}\right\}$ & & & &
\end{tabular}

Notice that we have not specified preferences of firms and workers completely. Any extension is permissible, subject to the preference orderings being consistent with the "good" sets specified above and the profile being in $\mathcal{P}_{S}$.

Note that the matching $\left\langle\left(F_{i},\left\{w_{1}, w_{2}, w_{3}\right\}\right) ;\left(F_{j},\left\{w_{4}, w_{5}\right)\right\rangle\right.$ is blocked by $\left\{F_{i}\right\} \cup$ $\left\{w_{2}, w_{4}, w_{5}\right\}$. To check this, note that $w_{2}$ is "good" for both $w_{4}$ and $w_{5}$. Moreover, $\left\{w_{2}, w_{4}, w_{5}\right\} \bar{P}_{2}\left\{w_{1}, w_{2}, w_{3}\right\}$, while $\left\{w_{2}, w_{4}, w_{5}\right\} P\left(F_{i}\right)\left\{w_{1}, w_{2}, w_{3}\right\}$. 
Consider $\left\langle\left(F_{i},\left\{w_{2}, w_{4}, w_{5}\right\}\right) ;\left(F_{j},\left\{w_{1}, w_{3}\right\}\right)\right\rangle$. This is blocked by $\left\{F_{i}\right\} \cup\left\{w_{2}, w_{3}, w_{4}, w_{5}\right\}$ since $w_{3}$ is "good" for $w_{i}, i=2,4,5$, and $\left\{w_{2}, w_{3}, w_{4}, w_{5}\right\} \bar{P}_{3}\left\{w_{1}, w_{3}\right\}$.

Also, $\left\langle\left(F_{i},\left\{w_{2}, w_{3}, w_{4}, w_{5}\right\} ;\left(F_{j},\left\{w_{1}\right\}\right)\right\rangle\right.$ is blocked by $\left\{F_{j}\right\} \cup\left\{w_{1}, w_{5}\right\}$ since $\left\{w_{1}, w_{5}\right\} P\left(F_{j}\right)\left\{w_{1}\right\},\left\{w_{1}, w_{5}\right\} \bar{P}_{5}\left\{w_{2}, w_{3}, w_{4}, w_{5}\right\}$ and $w_{5}$ is "good" for $w_{j}$.

Finally, it can be checked that $\left\langle\left(F_{i},\left\{w_{1}, w_{5}\right\}\right) ;\left(F_{j},\left\{w_{2}, w_{3}, w_{4}\right\}\right)\right\rangle$ is blocked by $\left\{F_{i}\right\} \cup\left\{w_{1}, w_{2}, w_{3}\right\}$. This is sufficient to show that $C(\mathbf{P})=\emptyset$.

\section{References}

1. V. Crawford and E.M. Knoer, Job Matching with Heterogeneous Firms and Workers, Econometrica 49 (1981), 437-450.

2. D. Gale and L. Shapley, College Admissions and the Stability of Marriage, American Mathematical Monthly 69 (1962), 9-15.

3. A. Kelso and V. Crawford, Job Matching, Coalition Formation, and Gross Substitutes, Econometrica 50 (1982), 1483-1504.

4. A. Roth, The Economics of Matching: Stability and Incentives, Mathematics of Operations Research 7 (1982), 617-628.

5. A. Roth, The College Admission Problem is not Equivalent to the Marriage Problem, J. Econ. Theory 36 (1985), 277-288.

6. A. Roth and M. Sotomayor, "Two-Sided Matching: A Study in Game-Theoretic Modeling and Analysis,". Econometrica Society Monographs No. 18, Cambridge University Press, Cambridge (England), 1990. 